\title{
Cardiac Rehabilitation after Coronary Artery Bypass Graft Surgery
}

\section{M.Sumathi, M.Sc (Nursing), Dr. V.Selvanayaki , PhD (Nursing)(Research Guide)}

PhD Research Scholar, Vinayaka Mission University, Salem. Research Guide:, Principal, Vinayaka Missions Annapoorana college of Nursing, Salem.

\section{Introduction}

Most people who have undergone coronary artery bypass surgery benefit from participating in a structured, comprehensive cardiac rehabilitation program. The potential benefits of rehabilitation include an improvement in heart function, a lowering of the heart rate at rest and during exercise, and a reduced risk of dying or developing complications from heart disease.

A cardiac rehabilitation program is a combination of activities designed to help patients recover from cardiovascular events such as myocardial infarction and to prevent further complications. Cardiac rehabilitation programs also help patients recover from surgical and interventional procedures, such as coronary artery bypass graft surgery, angioplasty and stenting.

\section{Major goals of cardiac rehabilitation programs}

- Curtailing the pathophysiologic and psychosocial effects of heart disease.

- Limiting the risk for reinfarction or sudden death.

- Relieving the cardiac symptoms.

- Reversing the atherosclerosis by instituting programs for exercise training, education, counseling, and modifying risk factors.

- Reversal of patient's activities to promote their well being.

- Enhancing the psychological status of the patients.

\section{Core components of Cardiac Rehabilitation program after Coronary Artery Bypass grafting}

1. Smoking cessation

2. Warm up \& cool down exercises

3. Cardiac walking

4. Control of high blood pressure and diabetes mellitus

5. Dietary management

6. Lipid management

7. Weight management

8. Managing stress

\section{Smoking cessation:}

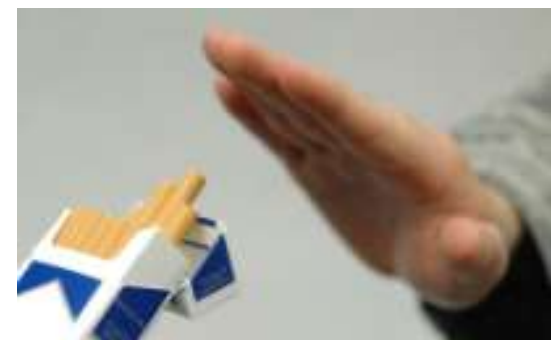

Smoking increases blood pressure, and affects the cardiac health very intensively. Quitting smoking is the first essential component of cardiac rehabilitation. When the patient has inability to stop smoking, he can get help from the health care professionals to get rid of this habit.

Once when the patient quit smoking, his blood pressure and heart rate becomes normal, breathing becomes easier and the level of oxygen and carbon dioxide becomes normal. 


\section{Warm up \& cool down exercises:}

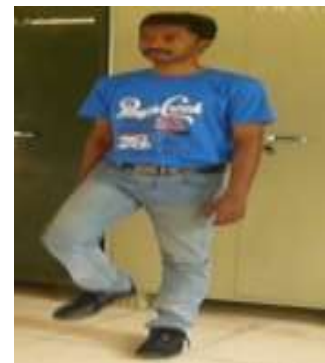

Exercise is also an important aspect in the cardiac rehabilitation program which helps in regulating optimal cardiac health. Before starting exercises certain precautions to be followed;

1. A large meal for at least 2 hours prior to the exercise session should be avoided.

2. Alcohol should not be consumed before exercise.

3. Drinking beverages with caffeine, i.e., tea, cola, etc. for at least 2 hours prior to the exercise to be avoided.

4. If the patient experiences any unaccustomed symptoms of pain, discomfort, or soreness, it should be informed to the nurse before starting the exercise.

5. Each exercise should be repeated 3-5 times and with the frequency of one to two times daily.

6. The warm up exercises should be done slowly and forceful movements to be avoided. The patient should never hold the breath and normal breathing is encouraged.

7. Good posture is important. The shoulders and back should be kept relaxed.

Types of exercises to be followed in Cardiac rehabilitation program;

\section{Marching}

- First the patient should start with simple marching for at least 3 minutes

2. Head and Neck exercises

3. Shoulder exercises

4. Arms exercises

5. Exercise for Trunk and Sides

6. Leg exercises

7. Quadriceps Stretch: Standing and Sitting

8. Hamstring Stretch: Standing and Sitting

All exercises have to be performed 10 times each. These exercises to be done slowly and should take 5 minutes to warm up and 5 minutes to cool down.

\section{Cool down}

Similarly, the patient should never speed up towards the end of his walk and then stop suddenly. It is important that he should gradually slow his pace during the last portion ideally 10 minutes of his exercise. This 'cool down' helps to reduce the risk of sudden heart problems or a sudden drop in blood pressure, and should be taken very seriously.

\section{Cardiac walking:}

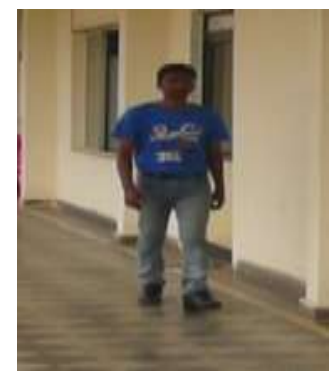

Walking is one of the most important exercise after surgery. It will improve the circulation, muscle tone, strength. Patient should be encouraged to walk every day, slowly increasing the distance.

\section{Home Walking Program}

1 st week walking should be started with three to five minutes, two to four times a day. 
2 nd week five to10 minutes, two to three times a day. If patient is tolerating this well, add one minute each day.

3 rd week 10 to 20 minutes one to three times a day.

$4^{\text {th }}$ week 15 to 25 minutes one to two times a day.

$5^{\text {th }}$ week 20 to 30 minutes one or two times a day.

$6^{\text {th }}$ week 25 to 35 minutes one time a day.

$7^{\text {th }}$ week 30 to 45 minutes one time a day.

$8^{\text {th }}$ week 45 minutes one time a day.

$9^{\text {th }}$ week onwards 45 minutes regularly one time a day.

\section{Control of high blood pressure and diabetes mellitus}

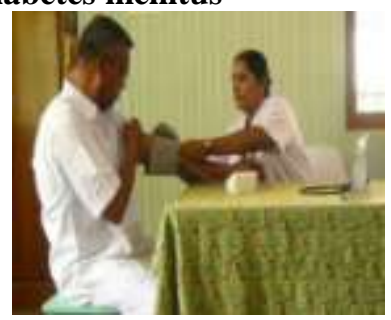

1. Too much sodium can aggravate high blood pressure by causing body to retain fluid. Avoidance of salt and high sodium foods such as pickles, papad, dry fish etc., is very essential.

2. Quitting smoking is important.

3. Checking blood pressure and blood glucose level regularly is necessary.

4. Prescribed medication should be taken regularly.

5. Maintaining ideal body weight is much important.

6. Recommended dietary pattern should be followed.

7. Avoidance of sweets and fatty foods are essential.

\section{Dietary management:}

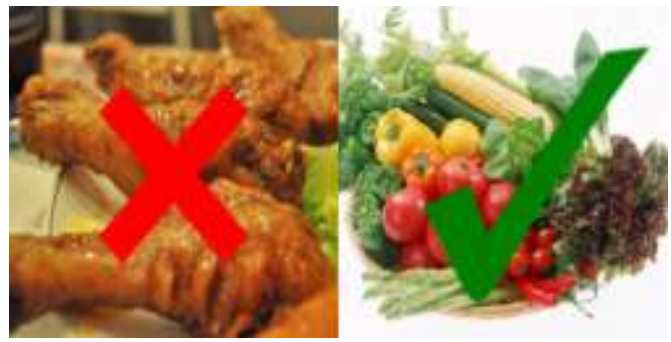

A good intake of Omega 3 fatty acid is important.

At least 5 portions of fruit and vegetables should be taken per day.

Reducing intake of saturated fat is necessary.

Restriction of salt intake is advised.

\section{Lipid management:}

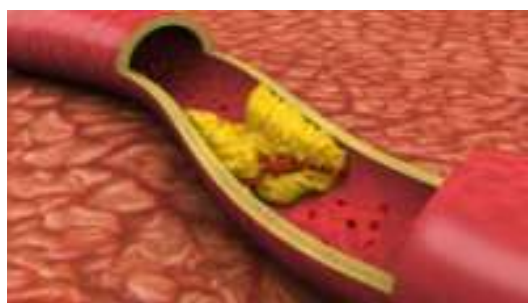

Cholesterol is a fatty substance made in the body. Cholesterol is derived from the food that we eat from animal sources, such as: eggs, butter, milk, cheese, meats. Our body requires certain amount of cholesterol; the excess cholesterol builds up along the inside lining of blood vessels. The total amount of cholesterol intake should be less than 200mg a day. Reducing the blood cholesterol by following the dietary guidelines is more important for cardiac patients. 


\section{Weight management:}

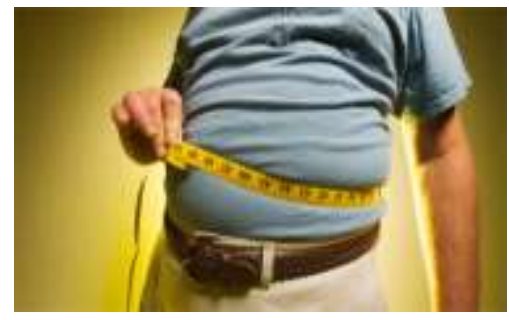

If the patient is overweight, losing weight will automatically help to reduce his risk of complications, and may also decrease cholesterol and blood pressure. To lose weight we need to eat less energy than our bodies use. Checking the weight often to know the baseline changes in the weight is considered much important.

\section{Managing stress}

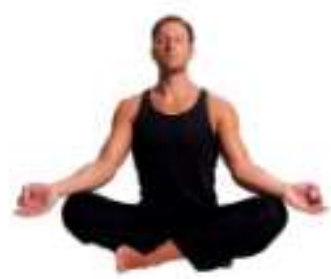

Stress is an important factor which leads to lot of cardiac complications and makes atherosclerotic changes in the blood vessels by inducing release of various hormones. Stress can be reduced by meditation, music therapy.

Meditation can be an effective form of stress reduction and has the potential to improve the quality of life. Meditation involves achieving a state of 'thoughtless awareness' in which the excessive stress producing activity of the mind is neutralized without reducing alertness and effectiveness.

Music therapy helps to promote Wellness, Manage Stress, Alleviate Pain, Express Feelings, Enhance Memory, Improve Communication, and Promote Physical Rehabilitation

\section{Conclusion}

Cardiac rehabilitation significantly improves functional capacity and some hemodynamic responses after post coronary artery bypass grafting. Therefore, patients need to participate in cardiac rehabilitation program to get fast recovery. Nurses have much important role in cardiac rehabilitation program.

\section{References}

[1]. American Heart Association, "Core Components of Cardiac Rehabilitation/Secondary Prevention Programs", Circulation, Dec2015 .

[2]. Vibhuti N Singh, "Cardiac Rehabilitation", The heart org.medscape, Aug 12, 2015.

[3]. Quinn R. Pack et.al., Participation in Cardiac Rehabilitation and Survival After Coronary Artery Bypass Graft Surgery- A Community-Based Study, The journal of Circulation, August 6, 2013.

[4]. Julian M Aroesty, Patient education: Recovery after coronary artery bypass graft surgery (CABG) (Beyond the Basics), Sep 2016. 